\title{
Fractal formation and ordering in random sequential adsorption
}

\author{
N. V. Brilliantov ${ }^{1,2}$, Yu. A. Andrienko ${ }^{1,3}$, P. L. Krapivsky ${ }^{4}$, and J. Kurths ${ }^{3}$ \\ (1) Moscow State University, Physics Department, Moscow 119899, Russia \\ ${ }^{(2)}$ Department of Chemistry, University of Toronto, Toronto, Canada M5S $1 A 1$ \\ (3) University Potsdam, Physics Department, Am Neuen Palais, D-14415 Potsdam, Germany \\ ${ }^{(4)}$ Courant Institute of Mathematical Sciences, New York University, New York, NY 10012-1185, USA
}

\begin{abstract}
We reveal the fractal nature of patterns arising in random sequential adsorption of particles with continuum power-law size distribution, $P(R) \sim R^{\alpha-1}, R \leq R_{\max }$. We find that the patterns become more and more ordered as $\alpha$ increases, and that the Apollonian packing is obtained at $\alpha \rightarrow \infty$ limit. We introduce the entropy production rate as a quantitative criteria of regularity and observe a transition from an irregular regime of the pattern formation to a regular one. We develop a scaling theory that relates kinetic and structural properties of the system.
\end{abstract}

PACS numbers: 02.50.-r, 05.40.+j

A variety of physical, chemical, and biological problems can be modeled by random sequential addition (RSA) processes [1,2]. Examples include adhesion of proteins and colloidal particles onto surfaces, polymer chain reactions, car parking, etc. The structure of disordered media, e.g. non-crystalline and granular materials, is also studied within the RSA-type model approaches (cf. [3, A1). In the simplest form, RSA processes can be formulated as sequential addition of objects that cannot overlap and, once inserted, cannot move or leave the structure.

Geometric and kinetic characteristics of RSA processes with identical objects are fairly well-known [1,2]. In contrast, adsorption of mixtures has been addressed in a very few studies [5 10]. If a mixture contains a small number of different sizes, geometric and kinetic characteristics are primarily determined by the smallest size. In some applications, e.g. for adsorption of latex spheres, or in modeling the structures of the coal grinds, ceramic or metallic powders, the size distribution may spread for several decades [3, 11]. Therefore, before the smallest size will finally win, an interesting intermediate asymptotics arises. To address this intermediate regime, we consider a continuous distribution with sizes ranging from zero up to some maximal size which we set equal to unity. For definiteness, we choose a power-law form

$$
P(R)= \begin{cases}\alpha R^{\alpha-1} & \text { for } R \leq 1 \\ 0 & \text { for } R>1\end{cases}
$$

for the rate of adsorption of particles with radii in the interval $(R, R+d R)(\alpha>0$ due to the normalization requirement). In the present Letter we show that RSA of particles with the size distribution (1i) gives rise to fractal structures with a fractal dimension that depends on the exponent $\alpha$. We also show that in the large $\alpha$ limit, highly regular structures arise which tend to the famous Apollonian packing as $\alpha \rightarrow \infty$.

Let $\Phi(t)$ is the fraction of uncovered area at time $t$ and $\Psi(R, t)$ is the probability that a disc of radius $R$ can be placed onto the surface, with a center at some arbi- trary point. The definition of $\Phi(t)$ and $\Psi(R, t)$ implies $\Psi(0, t)=\Phi(t)$ and

$$
\frac{d \Phi}{d t}=-\int_{0}^{\infty} d R P(R) \Psi(R, t) \Omega_{d} R^{d}
$$

Eq. (2) is written for the general case of adsorption onto a $d$-dimensional substrate, and $\Omega_{d}$ denotes the volume of the $d$-dimensional unit ball.

For the size distribution (11) one can assume a scaling behavior for the function $\Psi(R, t)$, i.e.

$$
\Psi(R, t)=S^{\theta} F\left(\frac{R}{S(t)}\right) .
$$

Here $S(t) \sim t^{-\nu}$ is a typical gap between neighboring adsorbed particles, and the scaling description should be relevant in the scaling regime, $t \gg 1$ and $R \ll 1$ with $R / S(t)$ finite. Without loss of generality we can set $F(0)=1$ and thus we get $\Phi(t)=S^{\theta}(t)$. Therefore, the scaling assumption for $\Psi(R, t)$ implies $\Phi(t) \sim t^{-z}$ with $z=\theta \nu$. Expressing Eq. (2) in the scaling form yields $\nu=(\alpha+d)^{-1}$ and $z \simeq \alpha \Omega_{d} \int_{0}^{\infty} d x x^{\alpha+d-1} F(x)$. Hence for $d=1$ we have $\nu=(1+\alpha)^{-1}$ in agreement with the exact result of Ref. [10], where it was justified that for the power-law distribution (11), the scaling behavior does appear in $1 \mathrm{D}$.

Now we relate the geometric (fractal) properties of the arising patterns with the kinetics. Let $n(R)$ be a number of adsorbed particles per unit area, with radii ranging from $R$ to $R+d R$. Clearly,

$$
n(R)=\int_{0}^{\infty} d t P(R) \Psi(R, t) \sim R^{\alpha-1+(z-1) / \nu}
$$

We can determine the fractal dimension $D_{f}$ of the pore space by introducing a cutoff size $\epsilon$, and calculating the number of particles per unit volume, $N(\epsilon)$, with radii greater than $\epsilon$. When this number behaves as a power law at small sizes limit, i.e., 


$$
N(\epsilon)=\int_{\epsilon}^{\infty} d R n(R) \sim \epsilon^{-D_{f}}
$$

one concludes that the fractal dimension is $D_{f}$ (see e.g. [12]). Notice that the porosity, i.e. the fraction of uncovered area, behaves as $\epsilon^{d-D_{f}}$. Combining Eqs. (位) and (5), we arrive to the following result for the fractal dimension:

$$
D_{f}=d-z(d+\alpha)
$$

Eq. (6) shows that the fractal dimension of arising patterns is intimately related to the exponent $z$, which describes the pattern formation kinetics. Similar relations between kinetics exponents and spatial properties of the patterns were observed for other pattern formation models [13,14]. The exact result for $z$ in 1D [10] completely solves the problem for the one-dimensional systems.

For $d \geq 2$ we could neither find the exact value of the exponent $z$, nor strictly prove the existence of scaling. To check the validity of the scaling theory, we performed numerical study of the 2D adsorption process. For every value of $\alpha$ we generated $10^{6}$ discs, and then computed the fractal dimension from the relation $N(\epsilon) \sim \epsilon^{-D_{f}} \quad 1214$. Typical patterns for small and large values of $\alpha$ are shown on Fig. 1 and Fig. 2, respectively. Fig. 3 gives the fractal dimension as a function of the exponent $\alpha$. We also present the value of fractal dimension, calculated from the scaling relation of Eq. (6), where numerical results for the kinetic exponent $z$ were used. Fig. 3 indicates that the scaling theory works fairly well in the whole range of $\alpha$.

The lack of evident spatial correlations at small $\alpha$ (see Fig. 1) suggests that a mean-field theory (MFT) can provide a reasonable approximation in this region. We develop a MFT similar to the one of Refs. [13, 14 and derived the following equation for the scaling function $F(x)$ :

$$
\begin{aligned}
& F\left(R t^{\nu}\right)= \\
& \exp \left\{-\int_{0}^{t} d \tau \int_{0}^{\infty} d \rho P(\rho) \Omega_{d}\left((R+\rho)^{d}-\rho^{d}\right) F\left(\rho \tau^{\nu}\right)\right\} .
\end{aligned}
$$

The ansatz $F(x)=\exp \left(-A_{1} x-\ldots-A_{d} x^{d}\right)$ solves Eq. (7) in arbitrary dimension. In particular, in 2D the coefficients $A_{1}$ and $A_{2}$ are determined from equations

$$
\begin{gathered}
A_{1}=2 \pi(\alpha+2) \alpha \int_{0}^{\infty} x^{\alpha} e^{-A_{1} x-A_{2} x^{2}} d x \\
A_{2}=\frac{\pi}{2}(\alpha+2) \alpha \int_{0}^{\infty} x^{\alpha-1} e^{-A_{1} x-A_{2} x^{2}} d x
\end{gathered}
$$

Solving Eqs. (8) and then inserting $F(x)=e^{-A_{1} x-A_{2} x^{2}}$ into expression $z=\alpha \pi \int_{0}^{\infty} d x x^{\alpha+1} F(x)$, derived previously, one finds $z$ and $D_{f}$. In particular, $D_{f}=2-\pi \alpha-$ $\left(\pi^{3 / 2}+\pi / 2\right) \alpha^{2}+\ldots$ in the small $\alpha$ limit. Mean-field results are given in Fig. 3. Fig. 3 indicates that the MFT works quite well for small $\alpha$, and it can be shown that MFT becomes exact at $\alpha \rightarrow 0$ limit. It is failed, however, for large $\alpha$, where the spatial correlations seem to be very important and the arising patterns strongly resemble the regular structures (see Fig. 2).

To quantify the increasing regularity of the structures, we introduce an entropy, $S_{N}$, characterizing the degree of order of $N$-particles patterns. If $C_{k}(N)$ denotes such a pattern and $p\left(C_{k}\right)$ denotes the probability of that pattern, one can define the Shannon entropy [15,16]:

$$
S_{N}=-\sum_{C_{k}} p\left(C_{k}\right) \log _{2} p\left(C_{k}\right)
$$

As it follows from the Eq. (9), $S_{N}=0$ for a regular pattern, since only one definite configuration with the probability $p=1$ contributes to the entropy. $S_{N}$ rapidly increases with increasing number of possible configurations, i.e. with decreasing order. The closely related value, $d S_{N} / d N \simeq S_{N+1}-S_{N}$ gives the entropy production rate and characterizes the regularity of the pattern formation process.

To find the entropy production rate, we first compute the conditional entropy $S_{N}\left[C_{k}(N-1)\right]$ of the $N$ discs for the given pattern $C_{k}(N-1)$ of $N-1$ discs. The conditional entropy is determined and calculated as follows: The $N$ th disc added to the pattern $C_{k}(N-1)$ can be treated as a point in the configuration $(x, y, R)$ space, where $x$ and $y$ are coordinates of the disc center, $R$ the disc radius. We divided the continuous configuration space into sufficiently small discrete cells and enumerated these cells (for computations, we used $\sim 10^{7}$ cells). Then, we introduced the probabilities $p_{i}$ that the next disc comes to the $i$ th cell for the given configuration $C_{k}(N-1)$, and calculated the conditional entropy as $S_{N}\left[C_{k}(N-1)\right]=-\sum_{i} p_{i} \log _{2} p_{i}$. The probabilities $p_{i}$ can be determined numerically for any given configuration $C_{k}(N-1)$ of $N-1$ discs. Namely, $p_{i}=$ $A_{i} R_{i}^{\alpha-1} / \sum_{j} A_{j} R_{j}^{\alpha-1}$, where $A_{i}=0$ if the disc corresponding to the $i$ th cell overlaps with some disk in the pattern $C_{k}(N-1)$, otherwise $A_{i}=1$.

The definitions of the conditional and full entropy then allows us to find the entropy production rate, $S_{N+1}-S_{N}$, by performing the averaging of the conditional entropy $S_{N}\left[C_{k}(N-1)\right]$ over all possible configurations $C_{k}(N-1)$ of $N-1$ disks. In practice, the averaging was performed over a number of $\left(\sim 10^{2}\right)$ Monte-Carlo runs.

To compare the entropy production rate for different values of $\alpha$, we plot $d S_{N} / d N$ as a function of the free area $\Phi$ (see Fig. 4). Fig. 4 shows the striking behavior of the entropy production rate at very large $\alpha$ : At the beginning of the process of pattern formation (i.e. at $\Phi \approx 1$ ), it decreases slowly in the same manner as for small $\alpha$, but at $\Phi \approx 0.55$ a sharp decay to a (small) plateau value is observed. One can interpret such type of behavior as a transition from a regime of "low regularity" to a regime of "high regularity" in the pattern formation process. The threshold value, $\Phi \approx 0.55$, is close to the jamming density, $\Phi_{\infty}=0.542 \ldots$, of the ordinary RSA of identical discs on a plane. The transition can be understood as follows: At the first "non-regular" stage of the process, 
random patterns mainly composed of discs with $R=1$ arise. After the jamming limit is achieved, the rule "insert the disc of maximal possible radius" starts to work [as it follows from Eq. (1) in the limit $\alpha \rightarrow \infty$ ], and much more regular patterns are produced. This transition corresponds to the sharp decrease of the entropy production rate observed in Fig. 4.

Now we argue that the fractal dimension of the patterns arising at the limit $\alpha \rightarrow \infty$ tends to that of the famous Apollonian packing, the oldest known fractal construction (see [17). To realize this we notice that in accordance with the rule "insert the disc of the maximal size", that works at $\Phi<\Phi_{\infty}$, the every new added disc touches (at least) three other ones. Therefore, after some time, all the free area will be scattered into a finite number (say $L$ ) of curvilinear triangles, confined by discs of radii $\left(a_{l}, b_{l}, c_{l}\right), l=1, \ldots, L$. Contribution to the free area from the other curvilinear polygons (confined by 4,5 , etc., touching discs) is negligible if time is large enough. Filling of these uncovered curvilinear triangles is performed in the very same way as in the Apollonian packing, namely, the every next disc placed touches from the inside the three outer touching discs. The fractal dimension of the pore space, obtained by the Apollonian filling does not depend on the starting set $\left(a_{l}, b_{l}, c_{l}\right)$ and equals to $D_{A}=1.305 \ldots$ 17. Thus we conclude that the fractal dimension of the patterns arising at $\alpha \rightarrow \infty$ coincides with that of the Apollonian packing.

In summary, we have investigated the adsorption kinetics and spatial properties of the arising patterns in a random sequential adsorption of particles with power-law size distribution. We have developed a scaling approach and verified scaling description by comparing with exact results in one dimension and numerical results in two dimensions. We have found that arising patterns have the fractal dimension $D_{f}$ that sensitively depends on the power-law exponent of the particles size distribution and is intimately related to the kinetic exponent of the time-dependent coverage. We have deduced that when $\alpha$, increases from 0 to $\infty, D_{f}$ decreases from 2 to $D_{A}=1.305 \ldots$, of the Apollonian packing. We have observed that the regularity of patterns increases with increasing $\alpha$ and introduce the entropy production rate as a quantitative criteria of the regularity.
[1] M. C. Bartelt and V. Privman, Int. J. Mod. Phys. 5, 2883 (1991).

[2] J. W. Evans, Rev. Mod. Phys. 65, 1281 (1993).

[3] S. V. Anishchik and N. N. Medvedev, Phys. Rev. Lett. 75, 4314 (1995).

[4] R. Jullien, P. Meakin, and A. Pavlovich, in Growth of Packings in Disorder and Granular Media, ed. D. Bideau and A. Hansen (Elsevier Sci. Publ. B.V., NY, 1993) p. 103.

[5] G. S. Baker and M. J. Grimson, Mol. Phys. 63, 145 (1987).

[6] J. Talbot and P. Schaaf, Phys. Rev. A 40, 422 (1989).

[7] M. C. Bartelt and V. Privman, Phys. Rev. A 44, 2227 (1991).

[8] P. Meakin and R. Jullien, Physica A 187, 475 (1992).

[9] B. Bonnier, Europhys. Lett. 18, 297 (1992).

[10] P. L. Krapivsky J. Stat. Phys. 69, 135, (1992).

[11] N. Standish, A. B. Yu, and R. P. Zou, Powder Technology 68, 175 (1991).

[12] S. S. Manna and H. J. Herrmann, J. Phys. A 24, L481 (1991)

[13] Yu. A. Andrienko, N. V. Brilliantov, and P. L. Krapivsky, J. Stat. Phys. 75, 507 (1994).

[14] N. V. Brilliantov, P. L. Krapivsky, and Yu. A. Andrienko, J. Phys. A 27, L381 (1994).

[15] M. C. Mackey, Rev. Mod. Phys. 61, 981 (1989)

[16] The summation over a continuous set of configurations implicitly assumes that we consider a finite subset of configurations; in a numerical implementation, this is clearly the case.

[17] B. B. Mandelbrot, The Tractal Geometry of Nature (San Francisco: Freeman, 1982).

Fig. 1 A typical pattern for $\alpha=0.1$. Only a small part of the total number of discs is shown.

Fig. 2 The same as in Fig. 1, $\alpha=50$.

Fig. 3 The fractal dimension $D_{f}$ versus $\alpha$. In the inset: kinetic exponent $z$ versus $\alpha$. The mean-field results are obtained from numerical soluion of Eqs. (8).

Fig. 4 The entropy production rate versus the uncovered area $\Phi$ for different values of $\alpha$. 


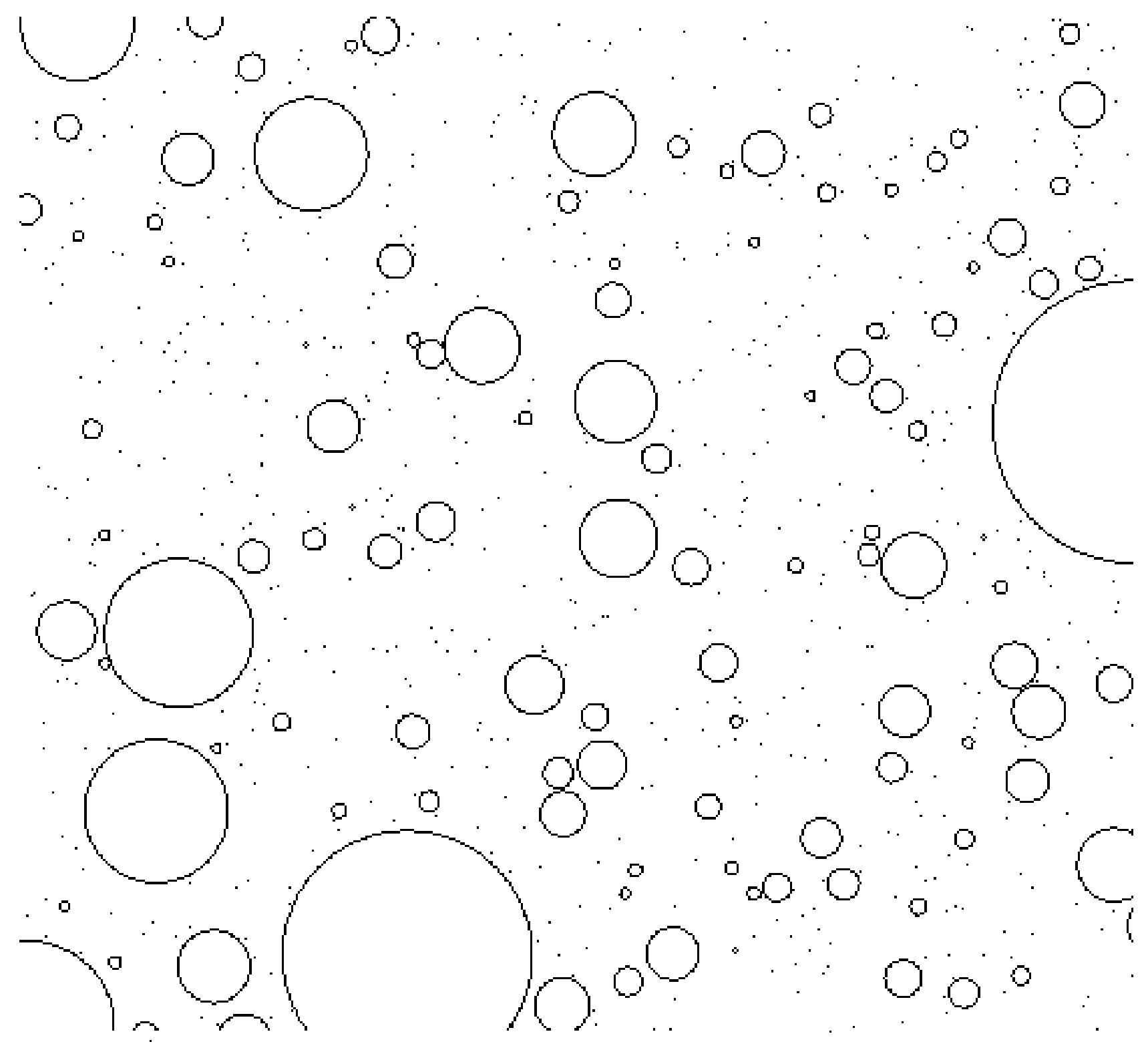




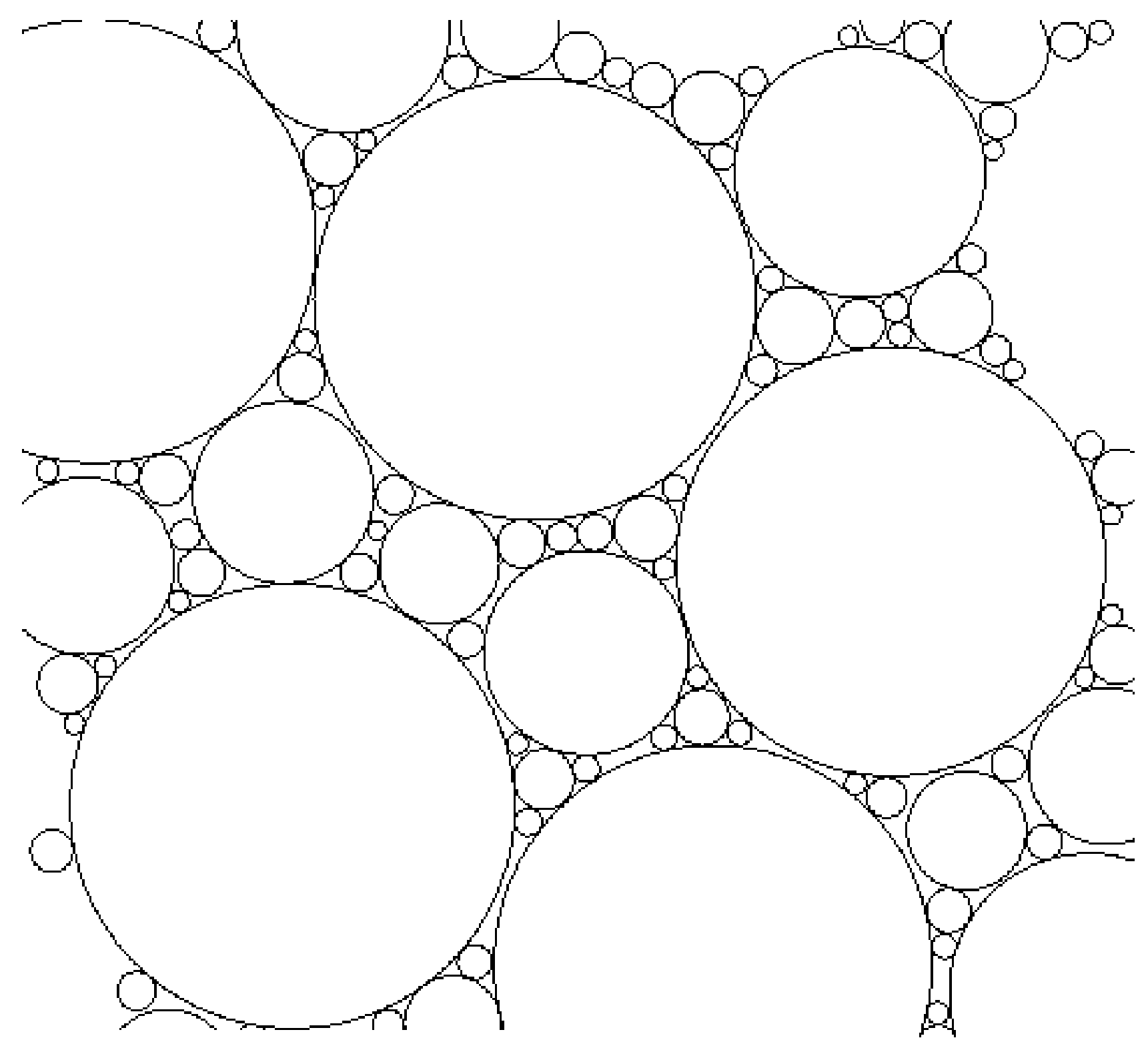




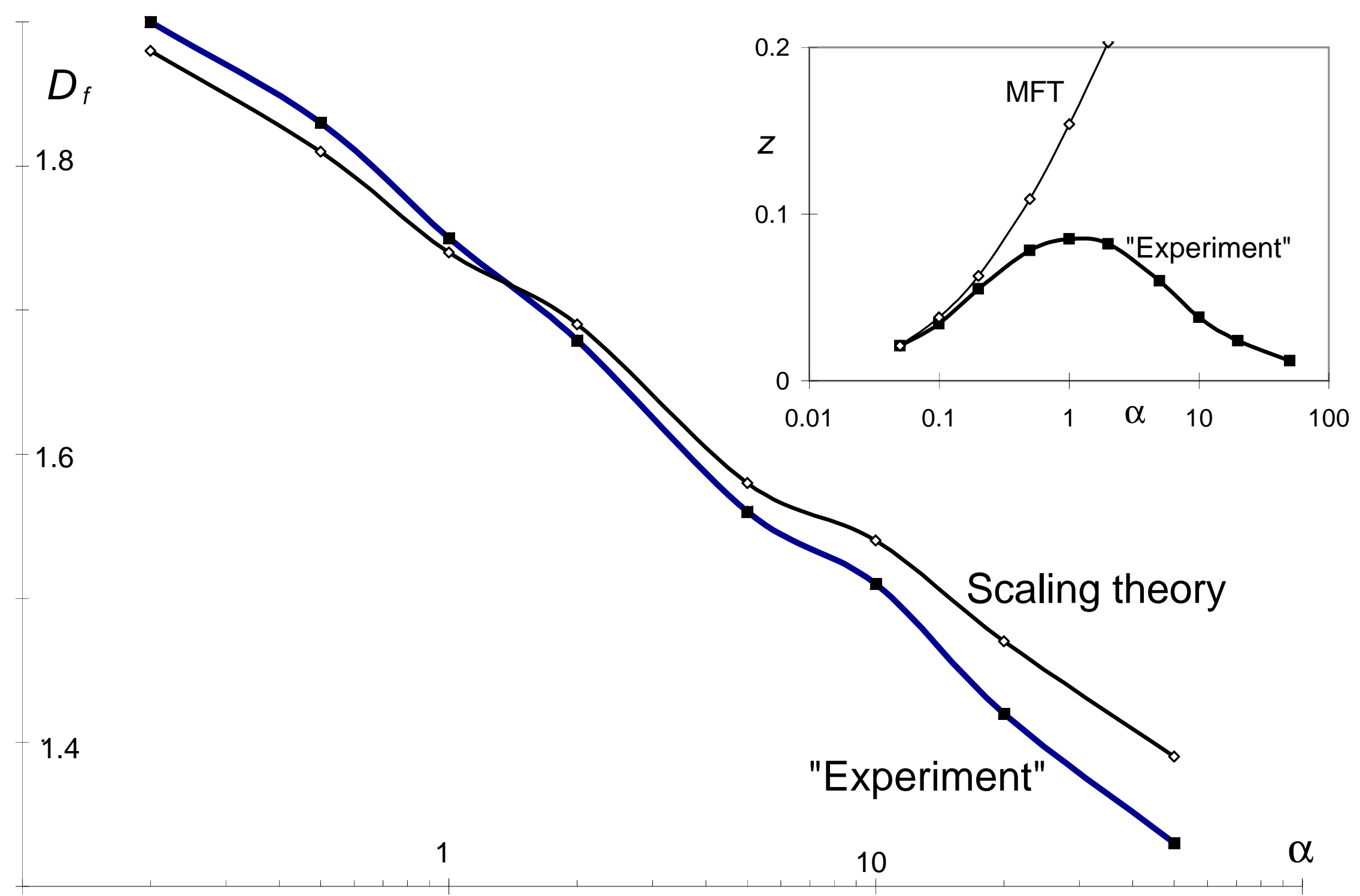




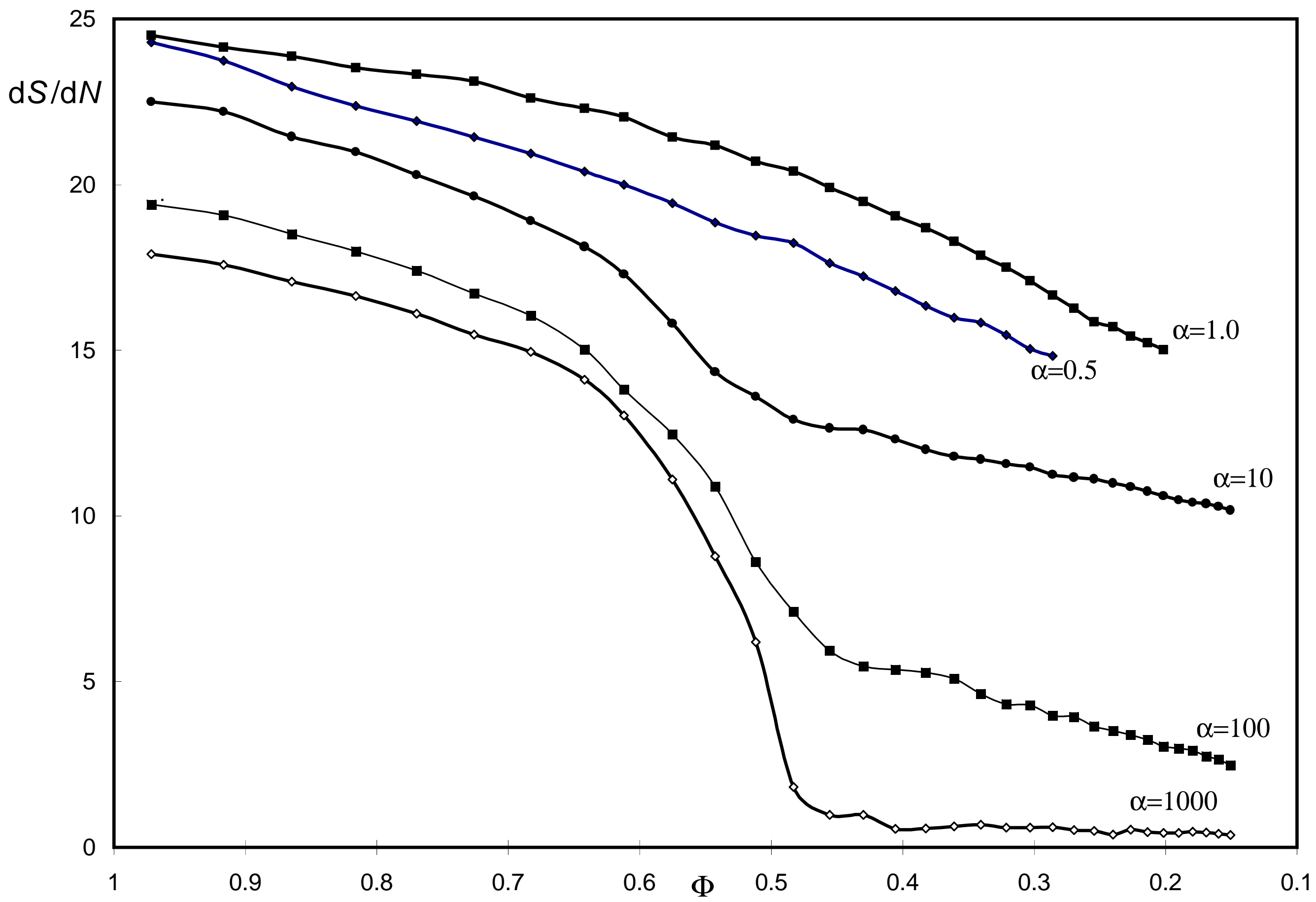

